\title{
Political topics (Fiction)
}

\section{AUTHOR}

Cordula Nitsch

\section{KEYWORDS}

entertainment, depiction of politics, political drama

\section{BRIEF DESCRIPTION}

The variable examines which political topics are prevalent in fictional entertainment. Studies differentiate either between the two categories political and sociopolitical issues (e.g., Eilders \& Nitsch, 2015) or they take a closer look at the presented political topics by differentiating between the three thematic dimensions of politics: polity for the institutional and normative infrastructure, policy for particular political issues, and politics for competition and power relations (e.g., Nitsch et al., 2021; Nitsch \& Eilders, 2015).

\section{FIELD OF APPLICATION/THEORETICAL FOUNDATION}

The differentiation between the three dimensions of politics goes back to political science and allows insights into which picture of politics is presented in the media. Analyses show that fictional (and non-fictional) media content tends to have a strong focus on the dimension of politics, i.e. movies and TV-series typically concentrate on negotiation processes between the parties and the power struggle between political camps (e.g., Jandura et al., 2016; Nitsch \& Eilders, 2015; Nitsch et al., 2021).

\section{REFERENCES/COMBINATION WITH OTHER METHODS OF DATA COLLECTION} $---$

\section{EXAMPLE STUDY}

Nitsch \& Eilders, 2015

\section{INFORMATION ON NITSCH \& EILDERS, 2015}

Authors: Cordula Nitsch \& Christiane Eilders

Research interest: depiction of politics (centrality of politics, topics, actors, political actions) in political dramas of two different countries (US and Germany)

Object of analysis: two political dramas ("The West Wing”, US; “Kanzleramt”, Germany)

Timeframe of analysis: 2004-2005

\section{INFORMATION ABOUT VARIABLE}

Variable name/definition: Politische Themen [political topics]

Pro Szene können bis zu drei politische Themen codiert werden, nämlich jeweils ein Thema aus den drei Bereichen polity, policy und politics. Eine Codierung von zwei Themen innerhalb eines der Bereiche (z.B. zwei policy Themen) ist NICHT möglich. In einer Szene, in der mehrere Themen durcheinander eingeworfen werden (z. B. eine Gruppe von Menschen redet durcheinander), muss folglich das zentrale Thema bestimmt werden. Lässt sich nicht bestimmen, welches das dominante Thema ist, wird das erstgenannte codiert.

Politics, policy, polity wird ausschließlich für den eigenen nationalen Kontext codiert. Außenpolitik wird als nationale policy codiert. Justizthemen werden meist im Bereich polity codiert; außer es wird Handlungsbedarf für die Politik artikuliert, dann werden diese Themen als Rechtspolitik bei den policies codiert.

[Up to three political topics can be coded per scene, namely one topic each of the three dimensions polity, policy and politics. The coding of two topics within one of the dimensions (e.g. two policy topics) is NOT possible. In a scene where several topics are addressed (e.g. a group of people talking), the central topic must be determi- 
ned. If it is not possible to determine which is the dominant topic, the first one is coded.

Politics, policy, polity is coded exclusively for its own national context. Foreign policy is coded as a national policy. Justice issues are usually coded in the area of polity; unless the need for political action is articulated, in which case these topics are coded as legal policy in the policies.]

Level of analysis: Szenenebene

Scale level: Nominal

Reliability: .83 (polity .70, policy .96, politics .84)

\section{V10 [THEMA-polity]} Polity (Strukturen und Institutionen)

$0 \quad$ kein polity Thema angesprochen

101 Bundesrat (USA: Senat)

102 Bundestag (USA: Congress)

103 Parlamente

104 Parteien bzw. Fraktionen (nur, wenn es um die Strukturen geht, aber nicht, wenn nur Parteien oder Fraktionen genannt werden!)

105 Regierung/ Regierungssysteme

106 Andere politische Institutionen

201 Internationale Richtlinien/ Internatio-

nale Abkommen und Regelungen

301 Gewaltenteilung

302

Föderalismus

Verfassung/-sgrundsätze / Zentrale Verfassungsprinzipien

Gesellschaftsordnung

Entscheidungsfindung/ Verfahrensord-

nung (sofern nicht ein Gremium bei den 100er Ausprägungen bezogen: Warum

gibt es einen Vermittlungsausschuss?

Regeln und Infrastruktur der Entscheidungsfindung --> Erklärungen über Abläufe)

Politische Kultur: Normen und Sitten (für die Stabilität des politischen Systems, z.B.: Sind Parteispenden Kavaliersdelikte...)

Bürokratie/ Verwaltung
Gerichte (Judikative)

Gesetze und Rechtsnormen/Grundgesetz Bürgerrechte

Menschenrechte

Öffentliches Recht (Völkerrecht, Staats-

recht, Sozialrecht usw.)

Strafrecht (Strafgesetzbuch)

Zivilrecht (Erbrecht, Familienrecht,
Schuldrecht usw.)

600 Polity in anderen Ländern

999 Sonstige Strukturen und Institutionen

\section{V11 [THEMA-policy]}

Policy (Politikbereiche)

Die Zuordnung einzelner Politikthemen zu den aufgeführten Bereichen ist kontextabhängig. So kann z.B. Drogenpolitik je nach Fokus unter Sozialpolitik, Arbeitspolitik oder Gesundheitspolitik verortet werden. Beim Thema Antisemitismus ist eine Zuordnung unter Diskriminierung möglich (wenn es um Antisemitismus allgemein geht) oder unter innere Sicherheit (wenn es um kriminelle Energie geht) oder unter Rechtspolitik (wenn ein Verfahren wegen antisemitischer Gewalt thematisiert wird).

$0 \quad$ kein policy Thema angesprochen

101 Außenpolitik (diplomatische Beziehungen, Konflikte usw.; Achtung: Hier wird nur das codiert, was nicht unter Verteidigungspolitik fällt; Außenpolitik wird auch codiert, wenn spezifische policies angesprochen werden, wie z. B. die Medienfreiheit in Ungarn oder die Bildungs politik in Italien)

102 EU-Politik (Institutionen, Erweiterung europäische Integration)

103 Entwicklungspolitik

104 Politik in anderen Ländern (nur, wenn keine Beziehung zu D bzw. USA)

105 Verteidigungspolitik (betrifft, ,äußere Sicherheit" wie Bundeswehr, NATO, Abrüs tung)

201 Innere Sicherheit (Terrorismus, Verfassungsschutz, Polizei, Links- und Rechtsradikalismus)

301 Bildungs- und Forschungspolitik (Hochschulpolitik, Lehrpläne, Technikentwick lung, ggf. Kindergärten-/Kindergartenknappheit --> sind je nach Kontext aber auch als Sozial- oder Familienpolitik denkbar)

302 Kulturpolitik (Film, Theater, Musik)

303 Medienpolitik (Meinungsfreiheit, Inhalte)

401 Sozialpolitik (Achtung: Sozialpolitik wird nur codiert, wenn keine Zuordnung zu detaillierteren Bereichen wie Familienoder Rentenpolitik möglich ist; ansonsten fallen hierunter z.B. Armut, Reichtum, Sozialabbau, Kinderarmut, Hartz4, 
keit)

402 Familienpolitik (Familie, Ehe, Scheidung, Erziehung, Kindergärten, Väterrechte)

403 Jugendpolitik

404 Rentenpolitik

501 Gesundheitspolitik

502 Drogenpolitik

503 Lebensmittelpolitik

504 Verbraucherschutzpolitik

601 Umwelt- und Klimapolitik

602 Energiepolitik

603 Agrar- und Forstwirtschaftspolitik

604 Infrastrukturpolitik (Wohnungsbau/Mietrecht/Eigentum, Städtebau, Post- und Fernmeldewesen)

605 Verkehrspolitik (Straßenbau, Schifffahrt, Luftfahrt, Schienenwege)

701 Arbeitspolitik (soziale Sicherung, Recht auf Arbeit, Tarife- und Lohnpolitik, Gewerkschaften, Arbeitsschutz, Arbeitnehmerrechte, Beschäftigungspolitik, Sexismus/sexuelle Belästigung am Arbeitsplatz, berufstätige Mütter, Diskriminierung am Arbeitsplatz, Arbeitslosigkeit) Wenn Wirtschafts- und Arbeitspolitik innerhalb einer Szene gleichwertig sind, wird Arbeitspolitik codiert.

702 Wirtschaftspolitik (Unternehmertum, Wirtschaftsförderung)

703 Finanzpolitik (Subventionen, Steuerpolitik, Haushalt, Geld- und Währungspolitik)

801 Rechtspolitik (Gesetzgebung, Grundrechte, Strafrecht, Kriminalität)

901 Migrationspolitik (Einwanderungspolitik, Ausländer- und Flüchtlingsfragen, Integrationsfragen, Migration)

902 Minderheitenpolitik (z.B. Diskriminierung, ethnische Minderheiten, Rassismus, Antisemitismus, religiöse Minderheiten, Homosexuelle, Menschen mit Behinderung) Asyl
V12 [THEMA-politics]

Politics (Wie wird etwas durchgesetzt? Diskussionen/Kämpfe; auch wenn es nicht um Parteienstreit im engeren Sinne geht, also auch bei außenpolitischen Verhandlungen)
0 kein politics Thema angesprochen

10 Abstimmungen/Entscheidungsverfahren (Darstellungen des Prozesses)

11 Ausschussarbeit

12 Parlamentsarbeit (große/kleine Anfragen, Anhörungen)

13 Gesetzgebungsverfahren

14 Gipfelkonferenzen

15 Untersuchungsausschuss

16 Sonstige politische Auseinandersetzungen

20 Bildung von Interessenskoalitionen

21 Interessenvermittlung/-artikulation/auswahl/-bündelung/-durchsetzung

22 Kampf um Entscheidungsbefugnis/ Legitimationsbeschaffung durch Verhandlungen

23 Koalitionsverhandlungen

24 Kompromisssuche/Konsensfindung

30 Lobbyismus/ Überzeugungsarbeit von außen

31 Öffentlichkeitsarbeit/ Pressekampagnen/ Instrumentalisierung von Medien

40 Personalentscheidung (Bewerbung um einen Posten, Entlassungen etc.)

41 Postenverteilung

50 Putsch/ Staatsstreich in anderen Ländern

60 Wahlen/Wahlkampf

61 Andere Partizipationsformen (Volksbegehren, Bürgerinitiative)

99 Sonstige Prozesse

\section{REFERENCES}

Eilders, C., \& Nitsch, C. (2015). Politics in Fictional Entertainment: An Empirical Classification of Movies and TV Series. International Journal of Communication, 9, 1563-1587.

Jandura, O., Gladitz, P., \& Nitsch, C. (2016). Parlamente in non-fiktionalen und fiktionalen Angeboten. Was man in „Berlin direkt“ und „Borgen“ über parlamentarische $\mathrm{Ab}$ läufe erfährt [Parliaments in non-fictional and fictional formats. What we learn about parliamentary procedures in "Berlin direkt" and "Borgen". Publizistik, 61(3), 287-304.

Nitsch, C. \& Eilders, C. (2015). Fictional politics on TV: Comparing the representations of political reality in the US-series "The West Wing" and the German series "Kanzleramt". Global Media Journal. German Edition, 5(1), 1-19.

Nitsch, C., Jandura, O., \& Bienhaus, P. (2021). The democratic quality of political depictions in fictional TV-entertainment. A comparative content analysis of the political drama 
Borgen and the journalistic magazine Berlin

direkt. Communications. The European Jour-

nal of Communication Research, 46(1), 74-94. 